\title{
New Hyperon Equation of State Table for Supernova Simulations and Neutron Stars.
}

\author{
Sarmistha Banik \\ BITS Pilani, Hyderabad Campus, Hyderabad-500078, India \\ E-mail: sarmistha.banik@hyderabad.bits-pilani.ac.in
}

Prasanta Char*

Astroparticle Physics \& Cosmology Division, Saha Institute of Nuclear Physics,

1/AF, Bidhannagar, Kolkata-700064, India

E-mail: prasanta.charesaha.ac.in

\begin{abstract}
The equation of state $(\mathrm{EoS})$ of hot and dense matter plays a fundamental role in the understanding of core-collapse supernova (CCSN). A phase transition from hadronic to exotic phases might occur in the early post-bounce phase of a CCSN. There are several EoSs, including quarks and hyperons, available for supernova simulations. However, none of them are compatible with the latest observational limit of $2 M_{\odot}$. Our EoS involving hyperon-hyperon interaction, for the first time, yields a $2.1 M_{\odot}$ cold, $\beta$-equilibrated neutron star (NS). It represents the first supernova EoS table involving hyperons that is directly compatible with the recent mass observation. We adopt a density-dependent relativistic mean field model and generate a full tabular equation of state of dense matter with $\Lambda$ hyperons for a broad range of density, temperature and charge-tobaryon number ratio. The model is exploited to describe uniform and non-uniform matter in a consistent manner. Further, light and heavy nuclei along with interacting nucleons are treated in the nuclear statistical equilibrium (NSE) model of Hempel and Schaffner-Bielich (HS) which includes excluded volume effects and relativistic interactions. Finally, the EoS is used to study the effect of hyperons on the black hole $(\mathrm{BH})$ formation.
\end{abstract}

7th International Conference on Physics and Astrophysics of Quark Gluon Plasma

1-6 February, 2015

Kolkata, India

${ }^{*}$ Speaker. 


\section{Introduction}

The life cycle of a star having mass many times higher than the sun usually ends with a rapid collapse of its Fe-core followed by a violent explosion, a supernova. These core-collapse supernovae (CCSN) are some of the most energetic events in our galaxy. After the collapse, a protoneutron star (PNS) of very hot and highly dense matter is born with trapped neutrinos. Its central density can reach a few times of the nuclear saturation density. Within a few seconds after the release of the neutrinos, the PNS generally evolves into a neutron star (NS) or a black hole (BH) depending on its mass. One of the most crucial physical input that governs this late evolution stage of the supernova remnant is the properties of dense matter of the PNS. Several relativistic and nonrelativistic EoSs of nucleons and nuclear matter $[1,2]$ have been suggested throughout the years. But presence of strange degrees of freedom in highly dense matter is an inevitability due to Pauli exclusion principle. So, many EoSs, suggested for supernova simulations, include quarks, hyperons and other exotic particles $[3,4,5]$. But, none of them satisfies the observational constraint of $2 M_{\odot}$ NS $[6,7]$.

Recently, we calculated the first realistic EoS table (BHB) including $\Lambda$ hyperons using density dependent relativistic hadron (DDRH) field theory model [8]. We considered only $\Lambda$ hyperons and excluded other hyperons due to lack of experimental data of their potential depth in nuclear matter. The presence of exotic particles can substantially change the dynamics of stellar collapse. In this paper we report the effects of hyperons on $\mathrm{BH}$ formation using the open source stellar evolution code $G R 1 D$ [9].

\section{Features of the new EoS}

Here we discuss the features of the BHB EoS tables. It consists of mainly two parts - the high density part and the low density part. The high density part describes matter well above nuclear saturation density using DDRH model in mean field approximation. Here, the baryonbaryon interaction is mediated by $\sigma, \omega$ and $\rho$ mesons. In addition to those, repulsive interaction between $\Lambda$ hyperons is mediated by $\phi$ meson. In the DDRH model, the coupling constants are density dependent and DD2 parameter set is used to calculate those couplings [10]. The DD2 model agrees very well with the nuclear symmetry energy properties from experiments and observations. The properties of DD2 model at saturation density can be found in [10]. Here, the hyperon-vector meson couplings are calculated from $S U(6)$ symmetry relations [11] and the hyperon-scalar meson coupling is estimated from hypernuclei data where the $\Lambda$ hyperon potential depth is taken $-30 \mathrm{MeV}$ in nuclear matter $[12,13]$. Matter at lower temperature and sub-saturation density consists of light and heavy nuclei coexisting with unbound nucleons. They are treated in extended nuclear statistical equilibrium (NSE) model where excluded volume effects and other medium effects are taken into account [14]. We treat electrons and positrons as a uniform background and add their contribution as a non-interacting ideal Fermi-Dirac in the EoS table. The contribution from neutrinos are not added because weak equilibrium is not attained in general. Now, to merge these two parts of the EoS, free energy per baryon is minimized at constant $T, n_{B}$ and $Y_{p}$ and a minimal hyperon mass fraction of $10^{-5}$ is introduced at the transition boundary. The BHB EoS table is constructed for a wide range of baryon density $\left(\sim 10^{3}-10^{15} \mathrm{~g} / \mathrm{cm}^{3}\right)$, temperature $\left(T=0.1\right.$ to $\left.10^{2.2} \simeq 158.49 \mathrm{MeV}\right)$ 
and proton fraction $\left(Y_{p}=0.01\right.$ to 0.6$)$. In this paper, we denote the BHB hyperonic EoS by BHB $\Lambda \phi$ and only nucleonic EoS by HS(DD2) [14]. We also impose $\beta$-equilibrium and charge neutrality condition on the supernova EoSs to study the properties of cold NSs.

\section{Numerical simulation of CCSN}

We use a spherically symmetric one dimensional general relativistic Eulerian hydrodynamic code $G R 1 D$ for the purpose of our simulation [9]. GR1D studies the systematics of stellar collapse to neutron stars and $\mathrm{BH}$ formation [15]. The code interpolates the original EoS table, to generate more data and check the thermodynamic consistency. Neutrino effects are very crucial in supernova simulations. Three neutrino species $v_{e}, \bar{v}_{e} \& v_{x}\left(=v_{\mu}, \bar{v}_{\mu}, v_{\tau}, \bar{v}_{\tau}\right)$ are considered in this code. The lepton fraction in the pre-bounce stage is parametrized as a function of density [16]. For the postbounce phase, this code uses a computationally efficient leakage and approximate heating scheme for neutrinos $[17,18]$. The leakage scheme provides approximate energy and number emission rates. Neutrino heating is included via a parameterized charged-current heating scheme. For a successful supernova explosion we increase the neutrino heating scale factor from 1 which is its normal value. For various progenitor models of Ref.[19] we performed the simulations. It has been argued that progenitor structure plays a greater role in the stellar collapse from PNS to BH than the details of neutrino treatment [15].

\section{Results and discussions}

First of all, we study the beta-equilibrated cold NS structure. We solve the TOV equation for static star and find the maximum mass for $\mathrm{HS}(\mathrm{DD} 2) \mathrm{EoS}$ is $2.42 M_{\odot}$, whereas for BHB $\Lambda \phi$ EoS, it is $2.1 M_{\odot}$. These values are consistent with the recent mass observations. Next we perform the CCSN simulation with GR1D using various nonrotating presupernova progenitor models [19]. We recently did an extensive comparative study of hyperon EoSs for supernova simulations [20]. In this paper we present the results for a $23 M_{\odot}$ progenitor with solar metallicity (s23WH07) [19] with neutrino heating factor, $f_{\text {heat }}=1$.

Fig. 1 shows the temporal evolution of the shock radius and the baryonic and gravitational masses of PNS after the core bounce, obtained from simulations. The hydrodynamic shock stalls after about $100 \mathrm{~km}$ and recedes into an accretion shock. The gravitational mass of the PNS goes beyond the static stable value due to continuous mass accretion. This leads to a prompt collapse of the PNS into a BH denoted by the spike in gravitational mass and also in the shock radius. The effect of hyperons can also be clearly seen in the plots. As the appearance of hyperons makes the EoS softer and leads to even faster collapse of the PNS. After the core bounce, the BH formation time for $\mathrm{HS}(\mathrm{DD} 2) \mathrm{EoS}$ is $1.511 \mathrm{~s}$, whereas for $\mathrm{BHB} \Lambda \phi \mathrm{EoS}$, it is only $0.879 \mathrm{~s}$.

In Fig. 2 we show the total neutrino luminosity as well as the $v_{e}, \bar{v}_{e}$ and $v_{x}$ luminosities for $\operatorname{HS}(\mathrm{DD} 2)$ (left) and BHB $\Lambda \phi$ (right) models. Neutrino emission stops earlier for BHB $\Lambda \phi$ EoS, and also hints for an early creation of $\mathrm{BH}$ due to appearance of $\Lambda$ hyperons. 

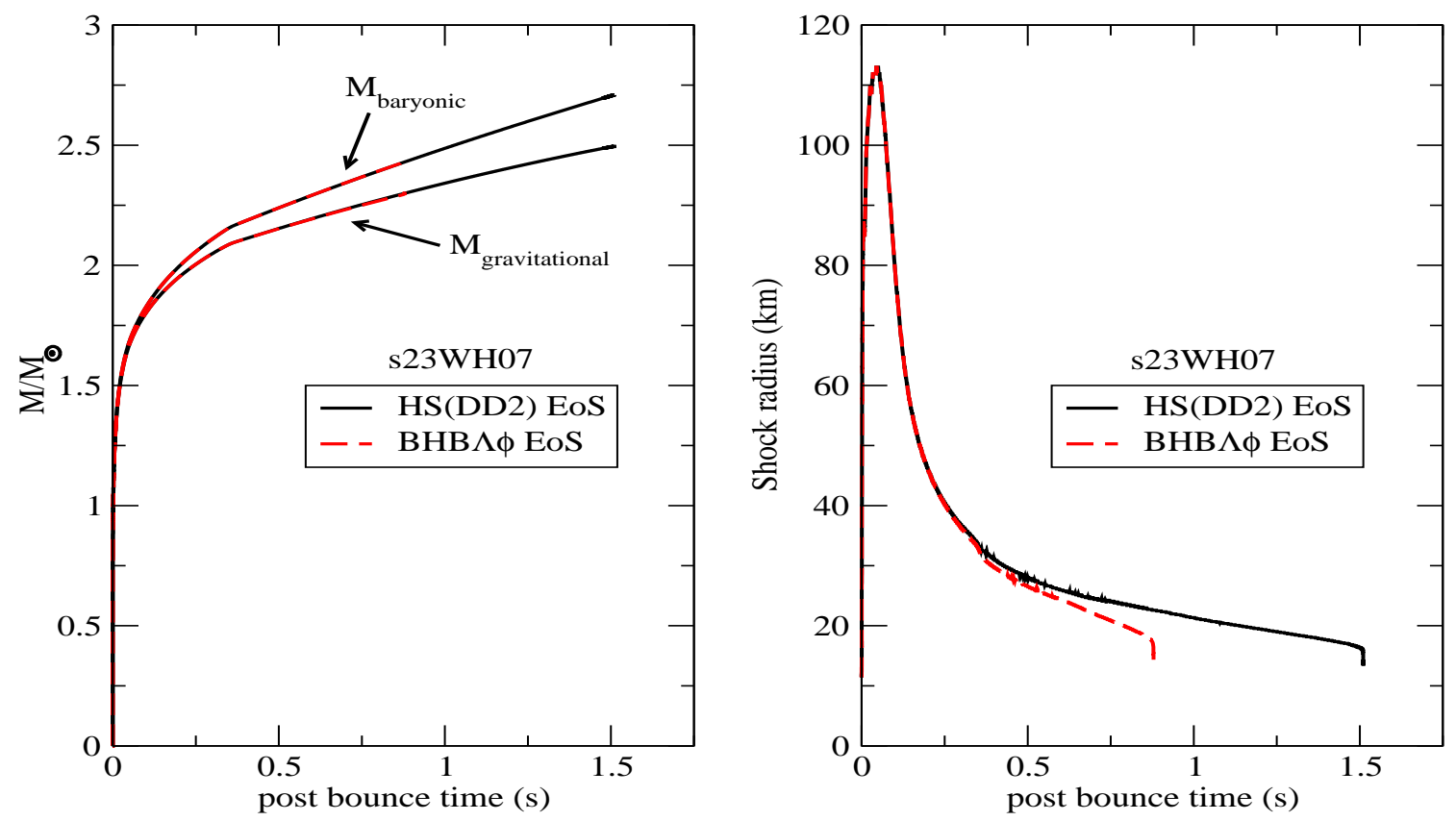

Figure 1: Post-bounce evolution of baryonic, gravitational masses (right panel) and shock radius (left panel) of the PNS for a $23 M_{\odot}$ progenitor model with $\mathrm{HS}(\mathrm{DD} 2)$ and BHB $\Lambda \phi$ EoSs.
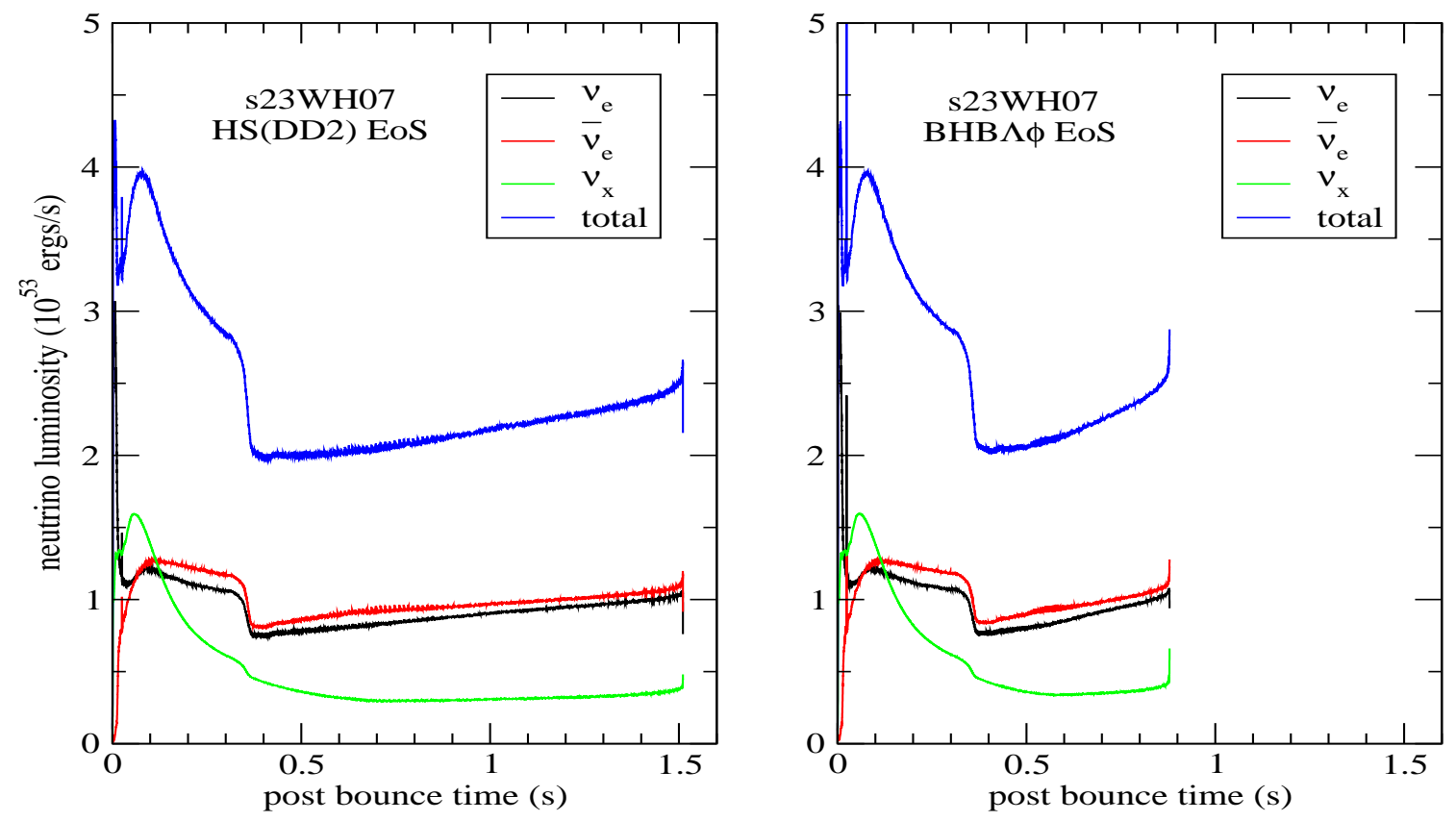

Figure 2: Total neutrino luminosity and $v_{e}, \bar{v}_{e}$ and $v_{x}$ luminosities and their evolution. 


\section{Summary}

We calculated a new hyperonic EoS within DDRH field theoretic model and applied it to study the effect of hadron-hyperon phase transition in CCSN simulations using GR1D code. We used a $23 M_{\odot}$ presupernova model and studied the formation and collapse of a PNS into a BH. We also confirmed that the emergence of hyperon triggers an accelerated collapse.

\section{Acknowledgements}

SB acknowledges the support from Department of Science \& Technology, India, BITS-Pilani, Hyderabad. PC acknowledges the support from Department of Atomic Energy, India.

\section{References}

[1] J. M. Lattimer, F. D. Swesty, Nucl. Phys. A, 535, 331 (1991).

[2] H. Shen, H. Toki, K. Oyamatsu, K. Sumiyoshi, Nucl. Phys. A, 637, 435 (1998).

[3] C. Ishizuka, A. Ohnishi, K. Tsubakihara, K. Sumiyoshi, S. Yamada, J. Phys. G, 35, 085201 (2008).

[4] M. Oertel, A. F. Fantina, J. Novak, Phys. Rev. C, 85, 055806 (2012).

[5] H. Shen, H. Toki, K. Oyamatsu,, K. Sumiyoshi, Astrophys. J. Suppl. Ser., 197, 20 (2011).

[6] J. Antoniadis et al. Science, 340, 1233232 (2013).

[7] P. B. Demorest, T. Pennucci, S. M. Ransom, M. S. E. Roberts, J. W. T. Hessels, Nature , 467 , 1081 (2010).

[8] S. Banik, M. Hempel, D. Bandyopadhyay, Astrophys. J. Suppl. Ser., 214, 22 (2014).

[9] C. D. Ott, E. O’Connor, Class. Quant. Grav., 27, 114103 (2010).

[10] S. Typel, G. Röpke, T. Klähn, D. Blaschke and H.H. Wolter, Phys. Rev. C , 81015803 (2010).

[11] J. Schaffner, I.N. Mishustin, Phys. Rev. C, 53, 1416 (1996).

[12] R.E. Chrien, C.B. Dover, Annu. Rev. Nucl. Part. Sci., 39, 113 (1989).

[13] C.B. Dover, A. Gal, Prog. Part. Nucl. Phys., 12, 171 (1984).

[14] M. Hempel, J. Schaffner-Bielich, Nucl. Phys. A, 837, 210 (2010).

[15] E. O’Connor, C. D. Ott, Astrophys. J., 730, 70 (2011).

[16] M. Liebendörfer, Astrophys.J. 633, 1042 (2005).

[17] S. Rosswog, M. Liebendörfer, MNRAS, 342, 673 (2003).

[18] H.-Th. Janka, A \& A, 368, 527 (2001).

[19] S. E. Woosley, A. Heger, Phys. Rept. 442, 269-283,(2007).

[20] P. Char, S. Banik, D. Bandyopadhyay, Astrophys. J. 809, 116 (2015). 